\title{
LINEAR PROPERTIES OF GOLDIE DIMENSION OF MODULES AND MODULAR LATTICES
}

\author{
EDMUND R. PUCZYŁOWSKI \\ Institute of Mathematics, University of Warsaw, 02-097 Warsaw, Banacha 2, Poland \\ e-mail:edmundp@mimuw.edu.pl
}

\begin{abstract}
We survey some old and recent results concerning the Goldie dimension of modules and modular lattices and its properties which are counterparts of properties of the dimension of linear spaces.
\end{abstract}

2000 Mathematics Subject Classification. Primary 16P60, $06 \mathrm{C05.}$

1. Introduction and preliminaries. The Goldie dimension of a module $M$, which is defined as the supremum of cardinalities $\lambda$ such that $M$ contains a direct sum of $\lambda$ nonzero submodules, is a generalization of the dimension of linear spaces (in short, linear dimension). The linear dimension can be characterized (or defined) in various ways. In many papers it was studied whether or how far these characterizations can be extended to the Goldie dimension. There were also many similar studies concerning the dual Goldie dimension.

The definition of the Goldie dimension and its main properties can be smoothly extended from modules to modular lattices with 0 . This more general context seems to be more appropriate for studying linear properties of that dimension, namely, some constructions available in the framework of lattices (e.g. lattices of ideals, factor lattices or some sublattices) help to describe such properties or distinguish lattices for which they are satisfied. Moreover, the results obtained for lattices apply directly to the Goldie and the dual Goldie dimensions of modules. It is worth to add that the idea of extending studies of some problems on rings and modules to modular lattices turned out to be fruitful also in some other situations (cf. $[\mathbf{1}, \mathbf{2}, \mathbf{1 5}]$ and also in the paper by T. Albu 'Goldie dimension, dual Krull dimension and subdirect irreducibility' in this issue).

Let $V$ be a linear space and $\operatorname{dim} V$ denote the dimension of $V$. This paper will concern extensions to the Goldie dimension of modules and modular lattices of the following properties of $\operatorname{dim} V$.

(1) $\operatorname{dim} V=\sup \{\alpha \mid V$ contains a direct sum of $\alpha$ nonzero subspaces $\}$.

(2) $\operatorname{dim} V=\operatorname{card} B$, where $B$ is an arbitrary basis of $V$. A basis of $V$ can be defined in many equivalent ways. The following two are most common:

(a) $B$ is a maximal linearly independent subset of $V$.

(b) $B$ is a minimal generating subset of $V$.

(3) If $\operatorname{dim} V=n<\infty$, then $\operatorname{dim} V$ is equal to the length $l(V)$ of $V$.

(4) $\operatorname{dim} V=n<\infty$ if and only if $V$ contains subspaces $W_{1}, \ldots, W_{n}$ such that $\operatorname{codim} W_{i}=1,1 \leq i \leq n, W_{1} \cap \cdots \cap W_{n}=0$ and this representation of 0 is irredundant.

Supported by Flemish-Polish bilateral agreement BIL2005/VUB/06 and Polish MNiSW Grant No. N N201 268435. 
(5). For arbitrary subspaces $W, T$ of $V$, the formula $\operatorname{dim}(W+T)+\operatorname{dim}(W \cap T)=$ $\operatorname{dim} W+\operatorname{dim} T$ holds.

Throughout the paper, $L$ stands for a modular lattice whose underlying partial order is denoted by $\leq$ and whose join and meet operations are denoted by $\vee$ and $\wedge$, respectively. We assume that $L$ has a least element 0 . If $L$ contains a greatest element, it is denoted by 1 .

For undefined concepts and fundamental results on modular lattices we refer to [11] and for those concerning modules we refer to [3].

For given elements $a \leq b$ of $L$, we denote by $[a, b]$ the interval $\{x \in L \mid a \leq x \leq b\}$.

Recall that modularity of $L$ means that for arbitrary $a, b, c \in L$, if $a \leq c$, then $(a \vee b) \wedge c=a \vee(b \wedge c)$ or, equivalently, for arbitrary $a, b \in L$, the map $x \rightarrow x \wedge b$ is an isomorphism of $[a, a \vee b]$ onto $[a \wedge b, b]$. The inverse isomorphism is $y \rightarrow a \vee y$.

The lattice dual to $L$ will be denoted by $L^{o}$. This lattice is modular as well.

For a given module $M$ the lattice of submodules of $M$ will be denoted by $L(M)$ and the lattice dual to $L(M)$ by $L^{o}(M)$. The lattices $L(M)$ and $L^{o}(M)$ are modular.

An ideal $I$ of $L$ is a non-empty subset of $L$ such that for arbitrary $a, b \in I,[0, a \vee$ $b] \subseteq I$. The set $I(L)$ of all ideals of $L$ is a complete, upper continuous modular lattice with respect to the partial order given by inclusion. Recall that a complete lattice $L$ is said to be upper continuous if for each $a \in L$ and every linearly ordered subset $X$ of $L$, $a \wedge(\bigvee X)=\bigvee_{x \in X}(a \wedge x)$

For a subset $S$ of $I(L), \bigwedge S=\bigcap S$ and $\bigvee S=\{x \in L \mid x \leq \bigvee F$ for a finite subset $F$ of $\bigcup S\}$.

The following lattice, which we denote by $T(a, b, c)$, will play a substantial role in several results presented in this paper. We call it the testing lattice. Saying that a lattice $L$ contains $T(a, b, c)$ or that $T(a, b, c)$ is a sublattice of $L$, we mean that there is a lattice embedding of $T(a, b, c)$ into $L$ which maps 0 of $T(a, b, c)$ onto 0 of $L$. In such a situation we will identify elements $a, b, c$ with their images in $L$.

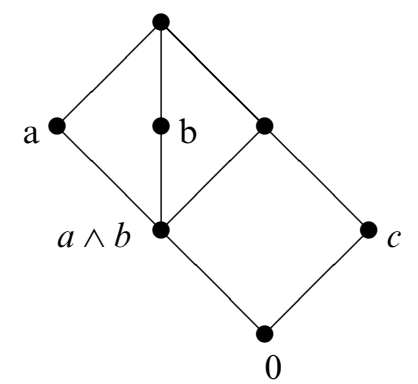

It turns out that in a number of cases the Goldie dimension of $L$ satisfies a given linear property if and only if $L$ does not contain the testing lattice with some extra properties of $a, b$ and $c$ as elements of $L$. Thus, the lattice $T(a, b, c)$ applies to test whether the Goldie dimension of a lattice satisfies some linear properties.

The following proposition expresses in module theory terms the fact that for a given module $M, L(M)$ contains the testing lattice.

Proposition 1. ([19]) For a given module $M$ and its nonzero submodules $A, C$ such that $A \cap C=0$, the following conditions are equivalent:

(1) $M$ contains a submodule $B$ such that the sublattice of $L(M)$ generated by $\{A, B, C\}$ is equal to $T(A, B, C)$;

(2) $A$ contains a nonzero submodule $K$ such that $A / K \simeq C$;

(3) There is a module epimorphism $f: A \rightarrow C$ with Kerf $\neq 0$. 

follows:

Modules $M$ such that $L^{o}(M)$ contains the testing lattice can be characterized as

Proposition 2. ([19]) For a given module $M$, the following conditions are equivalent:

(1) $L^{o}(M)$ contains the testing lattice;

(2) A homomorphic image $M^{\prime}$ of $M$ contains nonzero submodules $A^{\prime}$ and $C^{\prime}$ such that $M^{\prime}=A^{\prime} \oplus C^{\prime}$ and $C^{\prime}$ contains a proper submodule isomorphic to $A^{\prime}$.

2. Fundamental notions and results on Goldie dimension. In this section we survey the most fundamental notions and results related to the Goldie dimension of lattices and modules. We start with the following well-known result.

Proposition 3. For arbitrary elements $x_{1}, \ldots, x_{n}$ of $L$, the following conditions are equivalent:

(1) If $S, T \subseteq\{1, \ldots, n\}$ and $S \cap T=\emptyset$, then $\left(\bigvee_{s \in S} x_{s}\right) \wedge\left(\bigvee_{t \in T} x_{t}\right)=0$;

(2) For every $1 \leq i \leq n, x_{i} \vee\left(\bigvee_{j \neq i} x_{j}\right)=0$;

(3) For every $2 \leq i \leq n, x_{i} \vee\left(\bigvee_{j<i} x_{j}\right)=0$.

A set of nonzero elements of $L$ whose all finite subsets satisfy the conditions of Proposition 3 is called independent.

It is clear that a set $\left\{N_{t} \mid t \in T\right\}$ of nonzero submodules of a module $M$ is independent as a subset of $L(M)$ if and only if the sum $\sum_{t \in T} N_{t}$ is direct.

Proposition 4. ([12]) A subset $X$ of nonzero elements of $L$ is independent if and only if $\{[0, x] \mid x \in X\}$ is an independent subset of $I(L)$.

The Goldie dimension of $L$, denoted in this paper by $G \operatorname{dim} L$, is defined as $\sup \{\operatorname{card} X \mid X\}$ and is an independent subset of $L$.

Thus, the definition of the Goldie dimension is a direct extension of the definition of the linear dimension as stated in the property I in the Introduction.

From Proposition 4 it easily follows that for arbitrary $L, G \operatorname{dim} L=G \operatorname{dim} I(L)$.

For a module $M, G \operatorname{dim}(L(M))$ coincides with the Goldie dimension, $G \operatorname{dim} M$, of $M$.

In [21] Varadarajan introduced the concept of corank of a module $M$ and in [12] it was shown that it is equal to $\operatorname{Gdim}\left(L^{o}(M)\right)$. We call $G \operatorname{dim}\left(L^{o}(M)\right)$ the dual Goldie dimension of $M$ and denote it by $G^{o} \operatorname{dim} M$.

One may ask if $G \operatorname{dim} L=\lambda$, then does $L$ contain an independent subset of cardinality $\lambda$ ? This is obviously so if $\lambda<\infty$. Applying Theorem 6 and Proposition 3 it is not hard to show that the answer is positive for $\lambda=\aleph_{0}$. However, for larger $\lambda$ the problem is much more complicated. It was solved in [8] for lattices of modules.

Remarks 1. It is clear that in Proposition 3 the implications $1 . \Rightarrow 2$. $\Rightarrow 3$. hold for all, not necessarily modular, lattices with 0 . However, in the general case these conditions are not equivalent. Hence, for general lattices one can introduce different notions of independence choosing one of the conditions from 1-3 to define independence of elements. Relations among the so obtained notions (and some other) were studied in [24].

2. The class of lattices with 0 for which Proposition 3 holds is strictly wider than the class of modular lattices. In $[\mathbf{1 6}, \mathbf{1 7}, \mathbf{2 3}]$ it was proved that Proposition 3 holds for 
lattices satisfying the quasi-identity

$$
(x \wedge y) \vee((x \vee y) \wedge z)=0 \Rightarrow(y \vee z) \wedge x=0 .
$$

In these papers it was shown that most of the results on the Goldie dimension (in particular those stated in this section) of modular lattices extend to the class of lattices satisfying this quasi-identity.

An element $a \in L$ is called essential in $L$ if for every $0 \neq x \in L, a \wedge x \neq 0$.

If $a \leq b$ are elements of $L$ and $a$ is an essential element of [0,b], then we say that $a$ is essential in $b$ and is denoted in shortly as $a \leq_{e} b$.

A nonzero element $u \in L$ is called uniform if every nonzero element from $[0, u]$ is essential in $u$.

It is not hard to check that $u$ is a uniform element of $L$ if and only if $[0, u]$ is an essential element of $I(L)$.

Clearly, a subspace $U$ of a linear space $V$ over a field $F$ is a uniform element of $L(V)$ if and only if $\operatorname{dim} U=1$ or, equivalently, $U \neq 0$ and $U=F u$ for every $0 \neq u \in U$.

The following two results are the most fundamental characterizations of modular lattices with the finite Goldie dimension.

THEOREM 5. ([12]) The following conditions are equivalent:

(1) $G \operatorname{dim} L=n<\infty$;

(2) L contains independent uniform elements $u_{1}, \ldots, u_{n}$ such that $u_{1} \vee \cdots \vee u_{n}$ is an essential element of $L$.

THEOREM 6. ([12]) GdimL $<\infty$ if and only if for every sequence $a_{1} \leq a_{2} \leq \cdots$ there exists $j$ such that for all $k \geq j, a_{j}$ is essential in $\left[0, a_{k}\right]$.

Let us observe that the set consisting of elements $\left\{u_{1}, \ldots, u_{n}\right\}$ appearing in Theorem 5.2 is a maximal independent subset of uniform elements of $L$.

The following result proved in [13] extends Theorem 5 to the infinite case.

THEOREM 7. ([13]) If $A$ is a maximal independent subset of $L$ and all elements in $A$ are uniform, then for every independent subset $B$ of $L, \operatorname{card} B \leq \operatorname{card} A$.

Every maximal independent subset of $L$ consisting of uniform elements is called a basis of $L$.

For a module $M$, basis of $L(M)$ will be called $G$-basis of $M$ and basis of $L^{o}(M)$ will be called $G^{o}$-basis of $M$.

It may happen that $L$ contains no basis or even no uniform element.

EXAMPLE 8 . One can easily check that for every ring $P$ with unity and the ring $R=\prod_{i=1}^{\infty} P_{i} / \bigoplus_{i=1}^{\infty} P_{i}$, where for each $i, P_{i}=P$, the module $R_{R}$ contains no uniform submodule and, consequently, no $G$-basis.

Proposition 9. ([12]) For a given lattice $L$, the following conditions are equivalent:

(1) L contains a basis:

(2) $I(L)$ contains a basis;

(3) For every nonzero element l of $L$ there is a uniform element $u$ of $L$ such that $u \leq l$.

Theorem 7 immediately implies the following:

COROLLARY 10. If L contains a basis, then its cardinality is equal to GdimL. 
If $\left\{U_{i}\right\}_{i \in I}$ is a set of uniform elements of $L(V)$, where $V$ is a linear space over a field $F$, then $U_{i}=F u_{i}$ for $0 \neq u_{i} \in U_{i}, i \in I$. Now $\left\{U_{i}\right\}_{i \in I}$ is an independent subset of $L(V)$ if and only if the set $\left\{u_{i} \mid i \in I\right\}$ is linearly independent over $F$. Hence, the characterization of $\operatorname{Gdim} L$ as the cardinality of basis of $L$ is an extension of the characterization of $\operatorname{dim} V$ given in property $2(a)$ in the Introduction.

3. Goldie dimension and minimal generating sets. In this section we describe lattices to which one can extend the characterization of bases of linear spaces as minimal generating subsets (property 2 (b) in the Introduction).

We say that a set $U$ of uniform elements of $L$ is a generating subset of $L$ if for every nonzero element $l \in L$, there is a finite subset $S$ of $U$ such that $l \wedge(\bigvee S) \neq 0$.

Generating subsets of $L(M)$, for a module $M$, will be called $G$-generating sets of $M$. Note that these are families $\mathcal{U}$ of uniform submodules of $M$ such that $\sum_{U \in \mathcal{U}} U \subseteq_{e} M$.

Generating subsets of $L^{o}(M)$ will be called $G^{o}$-generating sets of $M$.

Note that $S$ is a $G$-generating set of a linear space $V$ over a field $F$ if and only if for some nonzero elements $v_{s} \in V, s \in S, S=\left\{F v_{s}\right\}$ and $\sum_{s \in S} F v_{s}=V$. This obviously holds if and only if the set $\left\{v_{s} \mid s \in S\right\}$ generates $V$ as a linear space. Consequently, minimal $G$-generating sets of $V$ correspond to bases of $V$ as characterized in 2 (b) in the Introduction.

Obviously, every basis of $L$ is a minimal generating subset of $L$. One may expect that, similarly as for linear spaces, the converse holds, i.e. every minimal generating subset of $L$ is a basis of $L$. This is not the case. Namely, $\{a, b\}$ is a minimal generating subset of the lattice $T(a, b, c)$ but it is not a basis of this lattice. The following example shows that there are modules without $G$-basis containing two-element $G$-generating sets.

EXAMPLE 11. (cf. [18]) Let $R=F[X]$ be the polynomial algebra over a field $F$ in continuum number of commutative indeterminates and $P=\prod_{\aleph_{0}} F / \bigoplus_{\aleph_{0}} F$. There is an $F$-algebra epimorphism $f: R \rightarrow P$. It induces an $R$-module structure on $P$. One can easily verify that $\{R \oplus 0,\{(r, f(r)) \mid r \in R\}\}$ is a minimal $G$-generating set of the $R$-module $M=R \oplus P$ and, applying Example 8, check that $M$ contains no basis.

The following result characterizes lattices in which minimal generating subsets are bases. It in particular shows that $T(a, b, c)$ can be used to test that property.

THEOREM 12. ([19]) For every L with a basis, the following conditions are equivalent:

(1) $U$ is a minimal generating subset of $L$ if and only if $U$ is a basis of $L$;

(2) If $u, v$ are uniform elements of $L$ such that $u \wedge v \neq 0$, then $u \vee v$ is a uniform element of $L$;

(3) L does not contain a sublattice $T(a, b, c)$ such that a is a uniform element of $L$.

Applying Theorem 12 and Proposition 1, one gets the following characterization of modules in which minimal $G$-generating sets are $G$-bases.

THEOREM 13. ([19]) For a given module $M$ which has a G-basis, the following conditions are equivalent:

(1) A set of uniform submodules of $M$ is a minimal $G$-generating set of $M$ if and only if it is a G-basis of $M$;

(2) For arbitrary uniform submodules $A, B$ of $M$ such that $A \cap B \neq 0$, the submodule $A+B$ is uniform; 
(3) $M$ fails to contain a direct sum $A \oplus C$ of nonzero submodules such that $A$ is a uniform module and $A / K \simeq C$ for a nonzero submodule $K$ of $A$.

Recall that a submodule $N$ of a module $M$ is called small if $N+T \neq M$ for every proper submodule $T$ of $M$ or, equivalently, $N$ is an essential element of $L^{o}(M)$. A nonzero module $M$ is called hollow if all proper submodules of $M$ are small or, equivalently, $M \neq 0$ and every proper submodule of $M$ is an essential element of $L^{o}(M)$.

Theorem 12 and Proposition 2 give the following result concerning $G^{o}$-generating sets and $G^{o}$-bases of modules.

THEOREM 14. ([19]) For every module $M$ which has a $G^{o}$-basis, the following conditions are equivalent:

(1) Every minimal $G^{o}$-generating set of $M$ is a $G^{o}$-basis of $M$;

(2) If $A, B$ are submodules of $M$ such that $A+B \neq M$ and $M / A, M / B$ are hollow modules, then $M / A \cap B$ is a hollow module;

(3) No homomorphic image of $M$ is a direct sum $A^{\prime} \oplus C^{\prime}$ of nonzero submodules such that $C^{\prime}$ is a hollow module and $A^{\prime}$ is isomorphic to a proper submodule of $C^{\prime}$.

It is well known and not hard to check that an abelian group is uniform if and only if it is a nonzero subgroup of the additive group of rational numbers or a cyclic or a quasi-cyclic $p$-group for a prime $p$.

Abelian groups satisfying Theorem 13 are completely classified.

THEOREM 15. ([19]) An abelian group $M$ satisfies conditions of Theorem 13 if and only if

(i) $M$ is a torsion-free group

or

(ii) $M$ is a torsion group such that for every prime $p$ the p-primary component of $M$ is one of the following groups: 0 , a direct sum of groups of order p, a cyclic p-group of order $\geq p^{2}$ or a quasi-cyclic p-group.

Not every minimal generating subset of $T(a, b, c)$ is a basis. However, the cardinality of every minimal generating subset of $T(a, b, c)$ is equal to its Goldie dimension. Thus, the class of lattices $L$ such that the cardinality of every minimal generating subset of $L$ is equal to $G \operatorname{dim} L$ is strictly wider than the class of lattices in which minimal generating subset are bases. The following theorem characterizes lattices of the finite Goldie dimension in the former of these classes.

THEOREM 16. ([19]) If L contains a finite generating subset, then the following conditions are equivalent:

(1) The cardinality of every minimal generating subset of $L$ is equal to $G \operatorname{dim} L$ (in particular $\operatorname{GdimL}<\infty$ ).

(2) For arbitrary uniform elements $u$, $v$ of $L, G \operatorname{dim}[0, u \vee v] \leq 2$.

(3) If $T(a, b, c)$ is a sublattice of $L$ and a is a uniform element of $L$, then $c$ is a uniform element of $L$.

Theorem 16 can be partially extended to arbitrary lattices containing generating subsets.

THEOREM 17. ([19]) If L contains a generating subset and for arbitrary uniform elements $u, v$ of $L, G \operatorname{dim}[0, u \vee v] \leq 2$, then $L$ has a basis and the cardinality of every minimal generating subset of $L$ is equal to $G \operatorname{dim} L$. 
Applying Theorems 16 and 17 one gets the following results for modules.

COROLLARY 18. ([19]) If a module $M$ has a $G$-generating set and for arbitrary uniform submodules $U, V$ of $M, G \operatorname{dim}(U+V) \leq 2$, then the cardinality of every minimal $G$ generating set of $M$ is equal to GdimM. If $M$ has a finite G-generating set, then the converse holds as well.

COROLlary 19. Let $M$ be a module which has a $G^{o}$-generating set. If for arbitrary submodules $U, V$ of $M$ such that the modules $M / U, M / V$ are hollow $G^{o} \operatorname{dim}(M / U \cap$ $V) \leq 2$, then the cardinality of every $G^{o}$-generating set of $M$ is equal $G^{o} \operatorname{dim}(M)$ (in particular $M$ has a $G^{o}$-basis). If $M$ has a finite $G^{o}$-generating set, then the converse holds as well.

Abelian groups satisfying Corollary 18 can be completely described as follows.

Proposition 20. ([19]) For a given abelian group $M$ and arbitrary uniform subgroups $U, V$ of $M, G \operatorname{dim}(U+V) \leq 2$ if and only if

(i) $M$ is a torsion-free group

or

(ii) $M$ is a torsion group

or

(iii) for precisely one prime $p$ the p-primary component of the torsion part of $M$ is nonzero.

The finiteness assumption in the second part of Corollary 18 is substantial even for abelian groups.

EXAMPLE 21 . Let $M=\bigoplus_{n=0}^{\infty} Z / n Z$, where $Z$ is the ring of integers. One easily checks that $\operatorname{Gdim} M=\aleph_{0}$ as well as the cardinality of every $G$-generating set of $A$ is equal to $\aleph_{0}$. However, by Proposition 20, $M$ contains uniform subgroups $U, V$ such that $\operatorname{Gdim}(U+V)>2$.

It would be interesting to describe rings $R$ such that all $R$-modules satisfy the conditions of Theorem 13 or Theorem 14, Corollaries 18 and 19, respectively. In [19] only basic relations among some of these classes of rings were established.

4. Goldie dimension and the length. The characterization of the linear dimension by the length (stated in property $\mathbf{V}$ in the Introduction) does not hold for the Goldie dimension of modules. For instance, $\operatorname{Gdim}_{Z} Z=1$ but $l(z Z)=\aleph_{0}$. It is a bit surprising that via some natural constructions one can find a relation between these invariants for modular lattices.

Define for $x, y \in L, x \sim y$ if and only if $x \wedge y$ is an essential element of $[0, x]$ as well as of $[0, y]$.

We have the following result.

Proposition 22. ([14], Proposition 2.1) is a congruence on the lower semilattice $(L, \wedge)$, i.e. $\sim$ is an equivalence relation on $L$ such that for arbitrary $x \sim y$ and $z \in L$, $x \wedge z \sim y \wedge z$.

For a given $x \in L$ denoted by $[x]$, the equivalence class of $L / \sim$ is determined by $x$. Define $[x] \leq[y]$ if and only if $x \wedge y \sim x$. It is not hard to check that so defined $\leq$ is a partial order on $L / \sim$. 
A lattice $L$ with 1 is called complemented if for every $a \in L$ there is $b \in L$ such that $a \wedge b=0$ and $a \vee b=1$ and it is called E-complemented [14] if for every $a \in L$ there is $b \in L$ such that $a \wedge b=0$ and $a \vee b$ is an essential element of $L$.

Obviously, every complemented lattice is $E$-complemented. It is not hard to check that the class of $E$-complemented lattices contains upper continuous lattices and lattices with the finite Goldie dimension.

THEOREM 23. ([14]) If $L$ is an E-complemented lattice, then $L / \sim$ is a modular complemented lattice.

For every $L, I(L)$ is an $E$-complemented lattice. Hence, Theorem 23 applies to $I(L)$. $L / \sim$.

The following result gives a relation between $G \operatorname{dim} L$ and the length of the lattice

THEOREM 24. ([14]) If GdimL $<\infty$, then $L / \sim$ is a modular complemented lattice and $\operatorname{Gdim} L=l(L / \sim)$.

5. Relations between Goldie and Kurosh-Ore dimensions. An element $a \in L$ is said to be irreducible in $L$ if $a \neq 1$ and for arbitrary $b, c \in L$ we have $a<a \wedge c$ whenever $a<b$ and $a<c$.

It is clear that a proper subspace $W$ of a linear space is an irreducible element of $L(V)$ if and only if $W$ is of co-dimension 1 .

The following classical result is referred as Kurosh-Ore theorem.

THEOREM 25. (Kurosh-Ore) If $a_{1} \wedge \cdots \wedge a_{n}=0=b_{1} \wedge \cdots \wedge b_{m}$ are irredundant representations of 0 in $L$ and all $a_{1}, \ldots, a_{n}, b_{1}, \ldots, b_{m}$ are irreducible elements of $L$, then $n=m$.

From Kurosh-Ore theorem it follows that the following notion is well defined.

Let $n$ be a positive integer. We say that the Kurosh-Ore dimension, $d(L)$, of $L$ is equal to $n$ if $L$ contains irreducible elements $a_{1}, \ldots, a_{n}$ such that $a_{1} \wedge \cdots \wedge a_{n}=0$ is an irredundant representation of 0 in $L$. If there is no positive integer $n$ such that $d(L)=n$, then we set $d(L)=\infty$. $d(M)$.

For a given module $M$ the Kurosh-Ore dimension of $L(M)$ will be denoted by

It is not hard to check that if $V$ is a finite dimensional linear space, then $\operatorname{dim} V=$ $d(L(V))$.

It turns out that the Kurosh-Ore and Goldie dimensions are closely connected and for modules they coincide.

THEOREM 26. ([12, 14]) If $d(L)=n<\infty$, then $\operatorname{Gdim} L=d(L)$.

One can easily find lattices $L$ with $G \operatorname{dim} L<\infty$ and $d(L)=\infty$. As an example (cf. [14]) one can take $L=L^{o}\left({ }_{R} R\right)$, where $R$ is the ring of integers localized at the multiplicative set generated by primes $\geq 5$.

A lattice $L$ is called a $*$-lattice if it is complete and for every linearly ordered subset $X$ of $L$ and every $a \in L,(\bigvee X) \wedge a \neq 0$ if and only if $x \wedge a \neq 0$ for some $x \in X$.

It turns out that for $*$-lattices Theorem 26 can be inverted.

THEOREM 27. ([14]) If $L$ is $a *$-lattice and $G \operatorname{dim} L<\infty$, then $G \operatorname{dim} L=d(L)$. 
Obviously, every upper continuous lattice is a $*$-lattice. Hence, examples of $*$-lattice are the lattice $I(L)$ of ideals of an arbitrary lattice $L$ and the lattice $L(M)$ of submodules of an arbitrary module $M$. Applying Theorem 27 one gets the following.

COROLlary 28.

(1) For every lattice $L$ with $G \operatorname{dim} L<\infty, G \operatorname{dim} L=G \operatorname{dim} I(L)=d(I(L))$.

(2) For every module $M, G \operatorname{dim} M<\infty$ if and only if $d(M)<\infty$. Moreover, $G \operatorname{dim} M=d(M)$.

Corollary 28 extends the characterization of $\operatorname{dim} V$ stated in property IV in the Introduction to the Goldie dimension of lattices and modules.

We conclude this section with a few remarks concerning the spanning dimension introduced by Fleury in [3] for modules.

We say that $L$ is an $F$-lattice if for every strictly increasing chain $a_{1}<a_{2}<\cdots$ of elements of $L$ there is an $n$ such that $a_{n}$ is essential in $L$.

From Theorem 6 it immediately follows that if $L$ is an $F$-lattice, then $\operatorname{Gdim} L<\infty$. Also note that every complete $F$-lattice is a $*$-lattice. Indeed, suppose $X$ is a linearly ordered subset of $L$ and $(\bigvee X) \wedge a \neq 0$ for some $a \in L$. If $X$ contains the greatest element $x$, then $\bigvee X=x$, so $x \wedge a \neq 0$. Otherwise $X$ contains a chain $x_{1}<x_{2}<\cdots$. As $L$ is an $F$-lattice, there is an $n$ such that $x_{n}$ is essential in $L$. Hence, $x_{n} \wedge a \neq 0$ and we are done. These observations and Theorem 27 give

COROLlary 29. If $L$ is a complete F-lattice, then $G \operatorname{dim} L=d(L)<\infty$.

In [3] Fleury considered modules such that (in our terminology) $L^{o}(M)$ are $F$ lattices. He rediscovered the Kurosh-Ore theorem in this case and called the obtained invariant, which in fact is $d\left(L^{o}(M)\right)$, the spanning dimension of $M$. Moreover, by the above corollary, $d\left(L^{o}(M)\right)=G^{o} \operatorname{dim} M$.

All results presented in this section concern finite dimensional lattices or modules. It would be interesting to study whether or how they can be extended to the infinite case.

6. Dimension modules and lattices. In [5] Camillo and Zelmanowitz observed that the formula stated in property $\mathrm{V}$ in the introduction does not extend to the Goldie dimension of modules. Modules which do satisfy this formula were called in [5] dimension modules. This notion can be easily extended to lattices.

A lattice $L$ is called a dimension lattice if for arbitrary $a, b \in L$ the following dimension formula is satisfied:

$$
\operatorname{Gdim}[0, a]+G \operatorname{dim}[0, b]=\operatorname{Gdim}[0, a \wedge b]+G \operatorname{dim}[0, a \vee b] .
$$

In this section we survey some results on dimension lattices and modules of the finite Goldie dimension. In infinite case many substantially new problems arise. In fact the studies in $[\mathbf{4}, \mathbf{5}]$ and later in $[\mathbf{2 2}]$ concerned finite dimensional modules (even the formula was stated in the form $G \operatorname{dim}(A+B)=G \operatorname{dim} A+G \operatorname{dim} B-G \operatorname{dim}(A \cap B)$, which is not very clear in infinite case).

In [5] the following characterization of dimension modules was obtained.

THEOREM 30. ([5]) A module $M$ fails to be a dimension module precisely when it has a submodule isomorphic to $X \oplus(X / Y)$ for some $Y \subseteq_{e} X$. 
This result is valid if $G \operatorname{dim} M<\infty$, but it does not hold without this assumption as the following example shows.

EXAMPLE 31. ([9]) Let $V$ be a linear space over a field $F$ such that $\operatorname{dim}_{F} V=\aleph_{0}$ and let $R=\left\{\left(\begin{array}{ll}f & v \\ 0 & f\end{array}\right) \mid f \in F, v \in V\right\}$. Note that $R$ is an $F$-algebra with respect to canonical matrix operations and there is an $F$-algebra epimorphism $\phi$ of $R$ onto $F$, which induces and $R$-module structure on $F$. Clearly $R$ is a local algebra so its radical $J$ is an essential $R$-submodule of $R$. Moreover, $\operatorname{Ker} \phi=J$, so $(R / J)_{R} \simeq F_{R}$. One easily checks that, because $J^{2}=0, R$-submodules of $R \oplus F$ contained in $J \oplus F$ are precisely $F$-subspaces of $R \oplus F$, so for such submodules the dimension formula holds. If an $R$-submodule $A$ of $R \oplus F$ is not contained in $J \oplus F$, then it contains $J \oplus 0$, so that $G \operatorname{dim} A_{R}=\aleph_{0}$. These show that the $R$-module $R \oplus F$ is a dimension module.

If in the above example $2 \leq \operatorname{dim}_{F} V<\infty$, then the $R$-module $M=R \oplus F$ is not a dimension module but the dimension formula is satisfied for uniform submodules of $M$ (cf. [19]). The question whether such examples exist was raised by del Valle in [22].

The following theorem collects some characterizations of dimension lattices.

THEOREM 32. For a given lattice $L$ with $\operatorname{GdimL}<\infty$, the following conditions are equivalent.

(1) $L$ is a dimension lattice.

(2) For arbitrary $a \leq_{e} b, c \in L$, we have $a \vee c \leq_{e} b \vee c$.

(3) $\sim$ is a lattice congruence on $L$.

(4) L does not contain the testing lattice $T(a, b, c)$ such that $a \wedge b \leq_{e} a$ (as elements of $L)$.

The equivalence of 1 and 2 for modules was proved by del Valle in [22] and for lattices in [18], where it was also proved that 2 and 3 are equivalent for arbitrary $L$. The equivalence of 1 and 4 was obtained in [9].

In [14] it was observed that $\sim$ is a lattice congruence for distributive lattices, lattices of non-singular modules and the lattice dual to the lattice of two-sided ideals of a unital ring.

7. Goldie dimension and matroids. A matroid $\mathcal{M}$ is defined as a pair $(S, \mathcal{P})$, where $S$ is a not-empty set and $\mathcal{P}$ is a collection of subsets of $S$ satisfying the following properties:

(1) $\emptyset \in \mathcal{P}$.

(2) If $X \in \mathcal{P}$ and $Y \subseteq X$, then $Y \in \mathcal{P}$.

(3) If $X, Y \in \mathcal{P}$ and $|X|=|Y|+1$, then there exists $x \in X \backslash Y$ such that $Y \cup\{x\} \in$ $\mathcal{P}$.

(4) If $A \subseteq S$ and every finite subset of $A$ is in $\mathcal{P}$, then $A$ is in $\mathcal{P}$.

$\mathcal{P}$ is called an independence space of $\mathcal{M}$ and sets of $\mathcal{P}$ are called independent subsets of $S$ or $\mathcal{M}$. A maximal with respect to inclusion set in $\mathcal{P}$ is called a basis of $\mathcal{M}$. It is known that cardinalities of all bases of $\mathcal{M}$ are equal. Their common cardinality is called the dimension of $\mathcal{M}$.

More information on Matroid Theory can be found in [20].

In [6] Dawson defined an independent space on the family of uniform submodules of a module and showed that its dimension is equal to the Goldie dimension of the module, provided the Goldie dimension is finite. Next, in [7] Dawson considered a dual 
independent space and an independent space connected with the Fleury dimension of modules [10] as well as its dual. Dowson's ideas and results were extended in [18].

Let $U(L)$ be the set of all uniform elements of $L$ (of course, it is possible that $U(L)=\emptyset)$ and let $\mathcal{P}$ be the set of all independent spaces in $L$ subsets of $U(L)$. In [18] it was proved that $(U(L), \mathcal{P})$ is a matroid. It is clear that every basis of $L$ is a basis of $(U(L), \mathcal{P})$ (the opposite implication does not always hold). In this way one can get as a consequence of results from Matroid Theory that the cardinality of every basis of $L$ (if it exists) is equal to $G \operatorname{dimL}$.

Applying Matroid Theory one can also get a relation between the Goldie dimension of $L$ and the length of a lattice related to the matroid $(U(L), \mathcal{P})$.

A subset $A$ of $U(L)$ is called a flat if for every independent in $L$ subset $K$ of $A$ and each $x \in U(L) \backslash A$ the set $K \cup\{x\}$ is independent in $L$.

THEOREM 1. ([18]) The set $F$ of flats of $U(L)$ is a modular lattice with respect to inclusion. If $G \operatorname{dim} L<\infty$, then $F \simeq L / \sim$, where $L / \sim$ is the lattice described in Section 3.

\section{REFERENCES}

1. T. Albu, M. Iosif and M. L. Teply, Dual Krull dimension and quotient finite dimensionality, J. Algebra 284 (2005), 52-79.

2. T. Albu and P. F. Smith, Localization of modular lattices, Krull dimension, and the Hopkins-Levitzki theorem II. Comm. Algebra 25 (1997), 1111-1128.

3. F. W. Anderson and K. R. Fuller, Rings and categories of modules (Springer Verlag, New York, 1974).

4. V. Camillo and J. Zelmanowitz, On the dimension of a sum of modules, Comm. Algebra 6 (1978), 345-352. 249-261.

5. V. P. Camillo and J. M. Zelmanowitz, Dimension modules, Pacific J. Math. 91 (1980), $29-36$.

6. J. E. Dawson, Independence spaces and uniform modules, Europ. J. Comb. 6 (1985),

7. J. E. Dawson, Independence structures on the submodules of a module, Europ. J. Comb. 6 (1985), 37-44.

8. J. Dauns and L. Fuchs, Infinite Goldie dimension, J. Algebra 115 (1988), 297-302. preprint.

9. P. Domagalska and E. R. Puczyłowski, Dimension modules and modular lattices,

10. P. Fleury, A note on dualizing Goldie dimension, Canad. Math. Bull. 17 (1974), 511-517.

11. G. Grätzer, General lattice theory (Birkhäuser Verlag, Basel, Switzerland, 1978).

12. P. Grzeszczuk and E. R. Puczyłowski, On Goldie and dual Goldie dimensions, J. Pure Appl. Algebra 31 (1984), 47-54.

13. P. Grzeszczuk and E. R. Puczyłowski, On infinite Goldie dimension of modular lattices and modules, J. Pure and Appl. Algebra 35 (1985), 151-155.

14. P. Grzeszczuk, J. Okniński and E. R. Puczyłowski, Relations between some dimensions of modular lattices, Comm. Algebra 17 (1989), 1723-1737.

15. P. Grzeszczuk and E. R. Puczyłowski, Gabriel and Krull dimensions of modules over rings graded by finite groups, Proc. Amer. Math. Soc. 105 (1989), 17-24.

16. J. Krempa, On lattices, modules and groups with many uniform elements, Algebra Discrete Math. 1 (2004), 75-86.

17. J. Krempa and B. Terlikowska-Osłowska, On uniform dimension of lattices, Contrib. Gen. Algebra 9 (Linz, 1994), 219-230 (Holder-Pichler-Tempsky, Vienna, 1995).

18. E. R. Puczylowski, On some dimensions of modular lattices and matroids, in International symposium on ring theory (Kyongju, 1999), 303-312 (Trends Math., Birkhauser Boston, Boston, MA, 2001).

19. E. R. Puczyłowski, A linear property of Goldie dimension of modules and modular lattices, submitted. 
20. D. J. A. Welsh, Matroid theory (Academic Press, London, 1976).

21. K. Varadarajan, Dual Goldie dimension, Comm. Algebra 7 (1979), 565-610. 1269.

22. A. del Valle, Goldie dimension of a sum of modules, Comm. Algebra 22 (1994), 1257-

23. A. P. Zolotarev, Balanced lattices and Goldie numbers in balanced lattices. Sibirsk. Mat. Zh. (Russian) 35 (1994), 602-611; translation in Siberian Math. J. 35 (1994), 539-546.

24. A. P. Zolotarev, On the interconnection among Helly, Radon and Caratheodory numbers in lattices with a balanced skeleton. Izv. Vyssh. Uchebn. Zaved. Mat. (11) (1992), 17-22 (1993); translation in Russian Math. (Iz. VUZ) 36(11) (1992), 15-20. 